\title{
An Analysis of a Staked Dipole Probe on a Lossy Earth Plane Using the Finite-Difference Time-Domain Method
}

\author{
David V. Thiel, Senior Member, IEEE, and Raj Mittra, Life Fellow, IEEE
}

\begin{abstract}
Using the finite-difference time-domain (FDTD) method, an electrically short, staked, grounded horizontal probe is shown to have an open circuit voltage directly related to the horizontal electric field at the earth's surface providing the surface coupling is subtracted from the output. This result is valid not only for an infinitely deep earth plane but also for one which is horizontally layered. The effective length of a staked probe is found to be dependent on the conductivity of the upper layer of the earth, its depth, the length of the stakes, and also the frequency of measurement. The effect is significant at very low frequency (VLF) for ice, permafrost and dry sand-covered regions.
\end{abstract}

Index Terms - Earth probes, electric field measurement, EM geophysics, FDTD, surface impedance.

\section{INTRODUCTION}

$\mathbf{S}$ INCE the 1950's when the use of surface impedance measurements was suggested for subsurface earth mapping [1]-[3], field researchers have employed a staked horizontal dipole probe (see Fig. 1) to measure the horizontal electric field component of an incoming radio signal [1]-[6]. More recently it was suggested that this method of measurement would result in the contamination of the electric field measurement with the magnetic field component present [7]. In the ensuing discussion [8]-[11], many points of argument were raised but the issue was not resolved.

Zonge and Hughes [12] demonstrated through measurement and calculation, that the effective length of a staked probe was dependent on the contact resistance between the probe and the ground. In the worst case, when the contact resistance was too high, the effective length of the probe was halved.

\section{THEORY}

For a vertically polarized plane wave source, the surface impedance $Z_{s}$ is defined by

$$
Z_{s}=\frac{E_{y}}{H_{x}}
$$

Manuscript received June 6, 1995; revised June 27, 1996. This work was conducted while D. Thiel was on a six-month sabbatical leave at the University of Illinois.

D. V. Thiel is with the School of Microelectronic Engineering, Griffith University, Queensland, Australia 4111 (e-mail: dthiel@me.gu.edu.au).

R. Mittra was with the Electromagnetic Communications Laboratory, University of Illinois, Urbana, IL 61801 USA. He is now with the Department of Electrical Engineering, Pennsylvania State University, University Park, PA 16802 USA (e-mail: r1mece@engr.psu.edu).

Publisher Item Identifier S 0196-2892(97)04105-3.

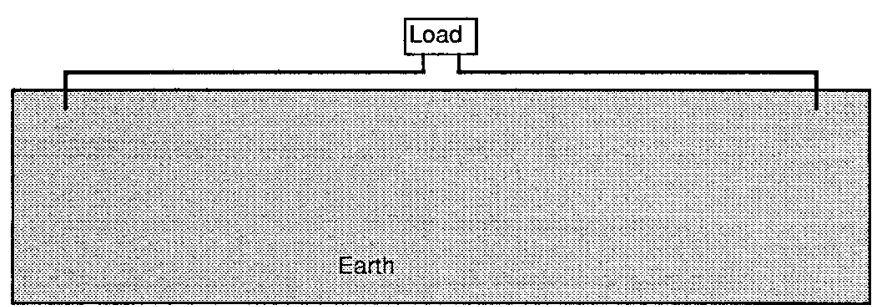

Fig. 1. Schematic representation of a staked electric field probe.

where $E_{y}$ is the horizontal electric field component and $H_{x}$ is the magnetic field component measured perpendicular to $E_{y}$. Both measurements are made on the surface of the earth. Clearly, if the measurement of $E_{y}$ is contaminated by an $H_{x}$ component, then the measured value of $Z_{s}$ would be in error [7].

Wait [8], [10] argued that one cannot separate the electric and magnetic field components in the far field, and suggested that the staked electric field probe monitors the magnetic field with an equivalent area given by:

$$
A=\frac{h}{\gamma}
$$

where $\gamma$ is the complex propagation coefficient in the lossy medium and $h$ is the separation distance between the stakes.

Note that

$$
\gamma=j \omega\left[\mu\left(\varepsilon+\frac{\sigma}{\delta \omega}\right)\right]^{1 / 2}
$$

where $\omega$ is the angular frequency of the radiation, $\mu$ is the magnetic permeability of the medium, $\sigma$ is the conductivity of the medium, $\varepsilon$ is the dielectric constant of the medium, and $j=(-1)^{1 / 2}$. Assuming that the conduction current dominates the displacement current for the earth material, one can rewrite (3) as

$$
A=\frac{h \delta}{1+j}
$$

where $\delta$ is the skin depth of the earth given by

$$
\delta=(\pi f \mu \sigma)^{-1 / 2}
$$

Thus, the loop antenna formed by the staked dipole has an area directly related to the skin depth of the radiation in the earth, and so is dependent on the angular frequency of the radiation and also the earth conductivity.

In the case of a thin wire loop, the open circuit voltage of an electrically small, single turn loop oriented in the plane 
perpendicular to the magnetic field $H$, is given by

$$
V_{o c}=j \omega \mu A H
$$

where $A$ is the area of the loop.

Returning to the loop formed by the staked antenna, combining (4) and (6) gives

$$
V_{o c}=(1+j)\left(\frac{\pi \mu}{\sigma}\right)^{1 / 2} f^{1 / 2} h H .
$$

It is interesting to observe that if $H_{x}$ is the magnetic field strength at the earth's surface, the product of the stake separation distance $h$, and the total magnetic field beneath the surface is given by

$$
\begin{aligned}
H_{x} A & =h \int_{0}^{\infty} H_{x} e^{-\gamma z} d z \\
& =\frac{-h H_{x}}{\gamma} .
\end{aligned}
$$

Thus, one can imagine the bounds of the subsurface loop lying between the two stakes which have infinite depth.

The voltage difference $V_{E}$ between two points, separated by a distance $h$ located parallel to a uniform electric field $E$, is given by

$$
\begin{aligned}
V_{E} & =\int_{0}^{h} E d h \\
& =h E .
\end{aligned}
$$

The open circuit voltage $V_{e o c}$ of an electrically small, elemental dipole having effective length $h_{e}$, located in air in the presence of a uniform parallel electric field $E$ is given by the expression:

$$
V_{e o c}=h_{e} E .
$$

The difference between (9) and (10) lies in the understanding that the current induced in the elemental dipole must be zero at its ends, and so, to a first order, will be triangular in shape along the dipole. Equation (9) is correct when the current distribution along a wire is uniform along its entire length. This is possible if the ends of the wire are terminated in a lossy earth plane.

Note that $V_{e o c}=V_{E}$ when

$$
\frac{E}{H}=\left(\frac{j \omega \mu}{\sigma}\right)^{1 / 2} \text {. }
$$

Equation (11) is an expression for the intrinsic impedance of the half space, and is equal to the surface impedance $Z_{s}$. Thus, we have a result which is self-consistent based on the assumption that (2) is correct, and is also consistent with [8]. Most important for this discussion is the concept that a staked antenna probe is a loop antenna having area $h / \gamma$ which is identical to an electric field probe of length $h$. The purpose of this paper is to investigate these relationships when the earth is nonuniform in the vertical direction.

In a recent paper [13], the FDTD was used to model the surface impedance over a variety of earth structures, although only the field values were calculated and not the voltage induced in wire probes. Both normal incidence and grazing incidence were found to give reliable surface impedance

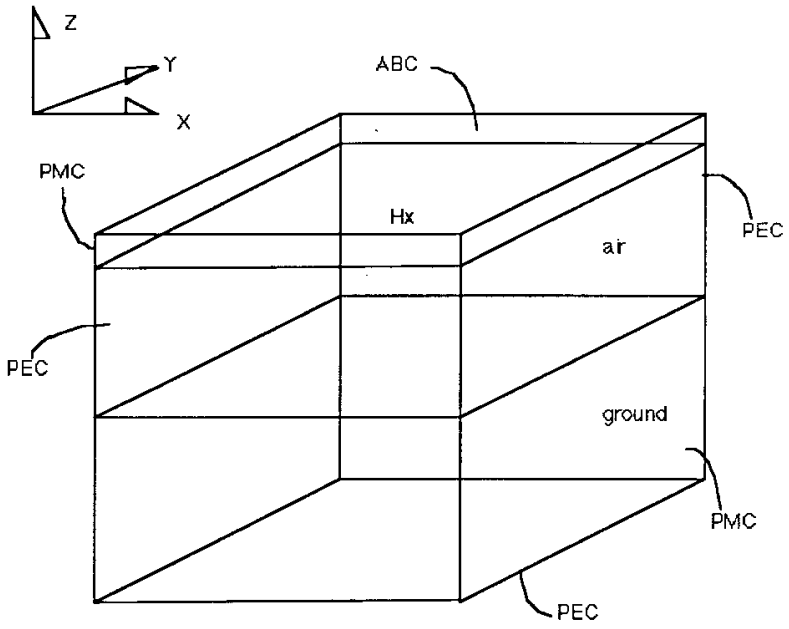

Fig. 2. FDTD solution space showing the coordinate system and the boundary conditions ( $n x=40, n y=40, n z=40$ ).

estimates. In this paper, we extend this model to investigate the voltage induced at the terminals of a number of probe configurations including a loop, a staked probe, and an insulated dipole. For maximum solution space, and to ensure the fields are horizontally uniform over the length of the probes investigated, only normal incidence is used.

\section{FDTD MODEL}

The three-dimensional solution space used in this analysis consisted of a $40 \times 40 \times 40$ array of cubic cells $(1 \times 1 \times$ $1 \mathrm{~m})$. The upper half of the space was air and included the magnetic field source $H_{x}$. Each model was run for 5000 time steps using the Courant stability condition [13] and a transient source excitation consisting of a Blackman-Harris window with a $10 \%$ cut-off frequency of $10 \mathrm{MHz}$. The coordinate system and the boundary conditions are shown in Fig. 2. The model was verified by calculating the surface impedance of the lossy earth (conductivity $0.01 \mathrm{~S} / \mathrm{m}$, depth $20 \mathrm{~m}$ ) above a perfect conductor (PEC boundary). Results are valid across the frequency range from $10 \mathrm{kHz}-1 \mathrm{MHz}$ with the magnitude of the surface impedance accurate to within $0.1 \%$ and the phase accurate to within less than $0.1^{\circ}$ [13]. This frequency range is convenient for this earth model, as at $1 \mathrm{MHz}$ the earth plane is effectively infinitely deep $(\delta=5 \mathrm{~m}$, so depth $=4 \delta$ ), and at $10 \mathrm{kHz}$ the earth plane is truly multi-layered $(\delta=50 \mathrm{~m}$, so depth $=0.4 \delta$ ).

The variation of $E_{y}$ with height and frequency is given in Fig. 3. Note that, at $1 \mathrm{MHz}, E_{y}$ increases by approximately $15 \%$ per meter. The variation in $H_{x}$ magnitude with height was found to be less than $1.5 \%$ over the first $5 \mathrm{~m}$ above the surface of the earth. The phase variation in $H_{x}$ was less than $1^{\circ}$ over the same distance. These results are consistent with electromagnetic wave reflection from a good conductor for both normal incidence [14] and also in the earth ionosphere wave guide [15] where the height gain function for the horizontal electric field approaches maximum rate of change and the height gain function for the horizontal magnetic field approaches minimum rate of change. 


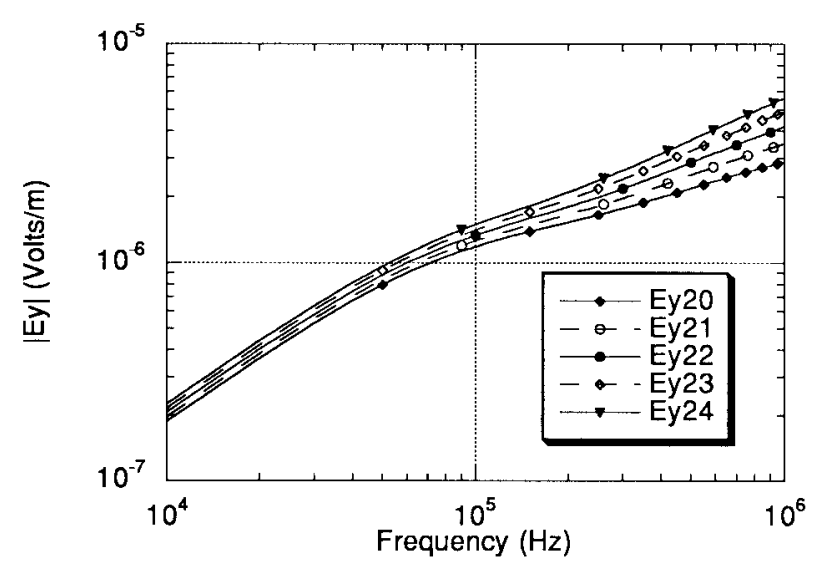

(a)

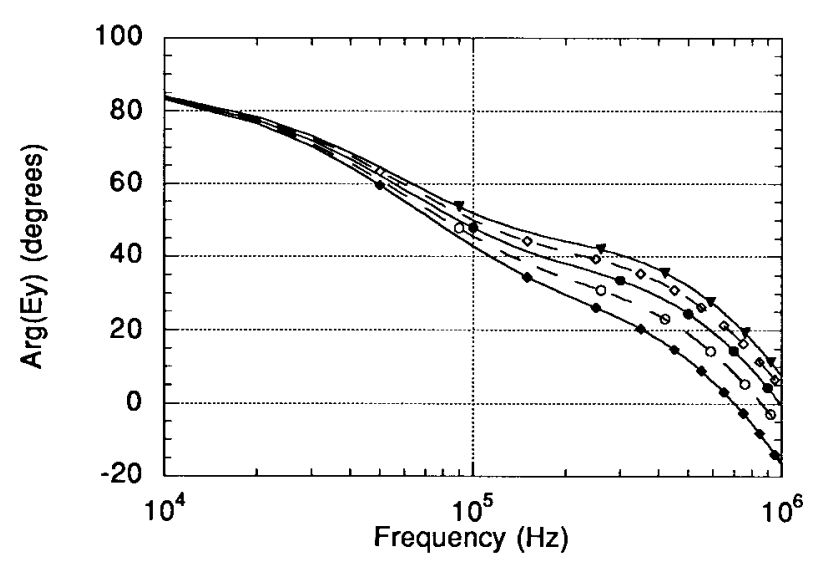

(b)

Fig. 3. Variation of $E_{y}$ with height (20-24 m) and frequency. Note that the surface of the earth is located at $z=20 \mathrm{~m}$.

Initial investigations were directed toward verifying the reception performance of two simple antenna structures; an electrically small conducting loop, and a symmetrical conducting horizontal dipole in air (Fig. 3). In order to remove concerns about input impedance, the feed point of these probes was a single air cell (i.e., zero conductivity and a relative permittivity of unity), and the voltage developed across the cell was treated as the probe output. While the probe feeds were oriented parallel to the $z$ axis, the three dimensional nature of the cells, and the rapid change of $E_{y}$ in the vertical direction, results in a significant potential drop across a single conductive cell in the $z$ direction. This results in some uncertainty in the position of the true feed point required to obtain agreement with theoretical results for infinitely thin wire structures.

\section{A. Elemental Loop}

An open circuit loop antenna with dimensions given in Fig. 4 was added to the three-dimensional (3-D) half space model, and the voltage across the open circuit was calculated. Results are given in Fig. 5, together with those derived from (6) using the magnetic field component also calculated from the FDTD solution. While the expectation was that the area of the loop was $3 \mathrm{~m}^{2}$, a much better fit is obtained using $4 \mathrm{~m}^{2}$. The reasons for this are unknown, but are thought to relate to

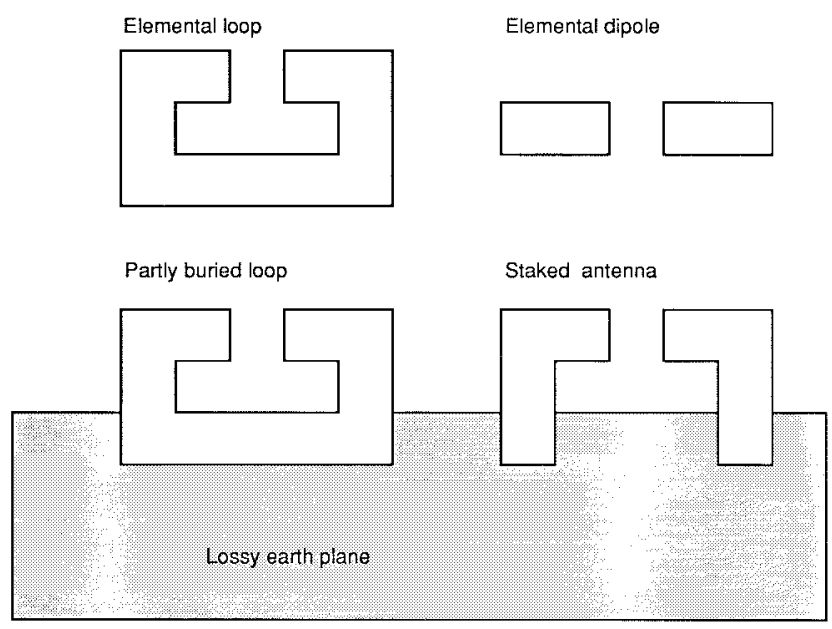

Fig. 4. Elemental field probes used in the FDTD model investigations: elemental loop antenna $5 \mathrm{~m} \times 3 \mathrm{~m}$ (11 cells); elemental dipole antenna 5 $\mathrm{m} \times 1 \mathrm{~m}$ (4 cells); partly buried loop $5 \mathrm{~m} \times 3 \mathrm{~m}$ (11 cells); and staked antenna $5 \mathrm{~m} \times 2 \mathrm{~m}$ ( 6 cells) above the surface. The length of stake below the surface of the earth is varied. Cell size is $1 \mathrm{~m} \times 1 \mathrm{~m} \times 1 \mathrm{~m}$.

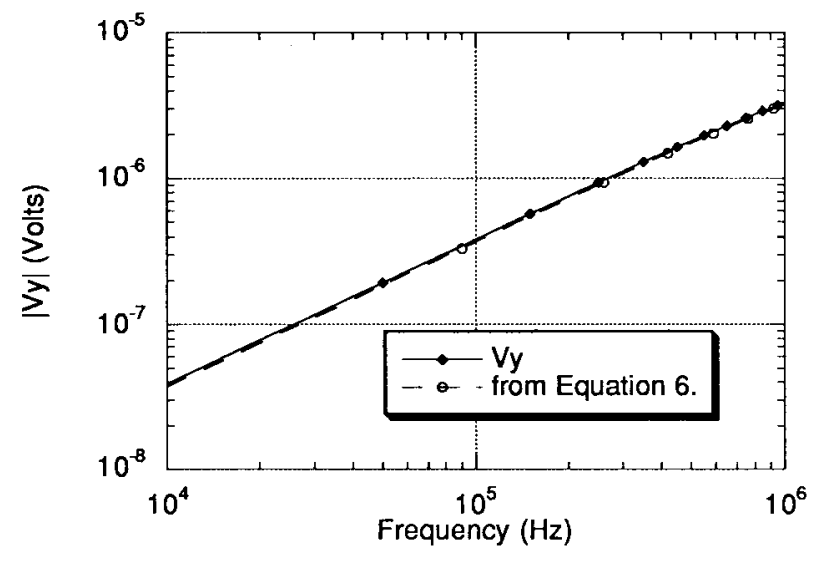

(a)

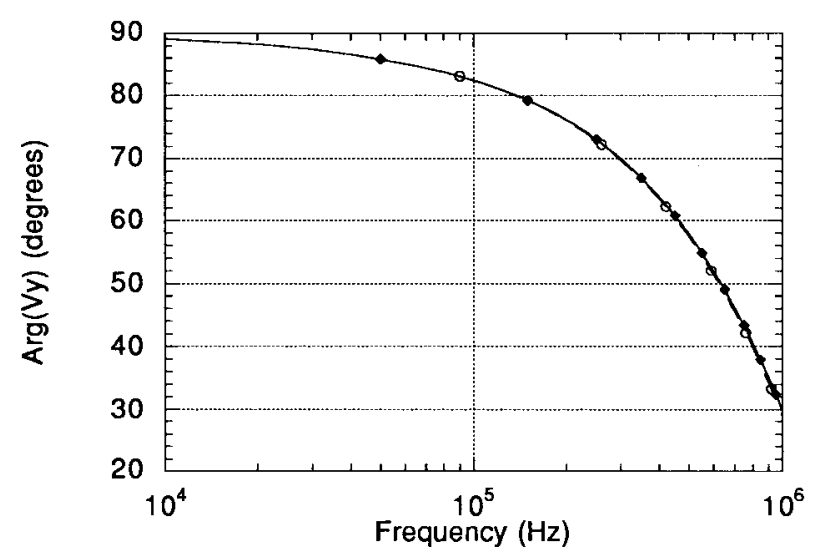

(b)

Fig. 5. FDTD result showing the open circuit voltage $V_{y}$ for the elemental loop antenna (continuous line) and that calculated from (6) (dotted line) using the magnetic field calculated from the half space FDTD model.

the large diameter wire used in the loop cell model. What is most important in this investigation is that the cell loop has the frequency characteristics of the magnetic field, not the electric field shown in Fig. 3. 


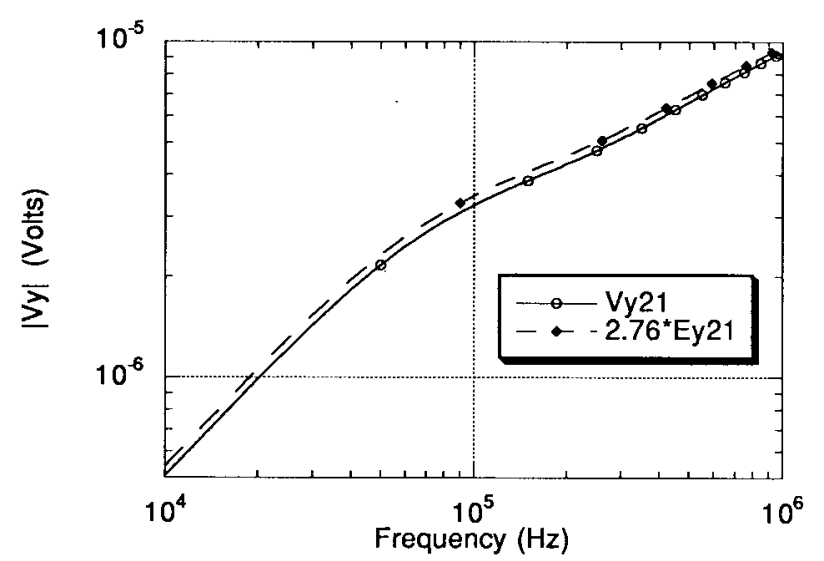

(a)

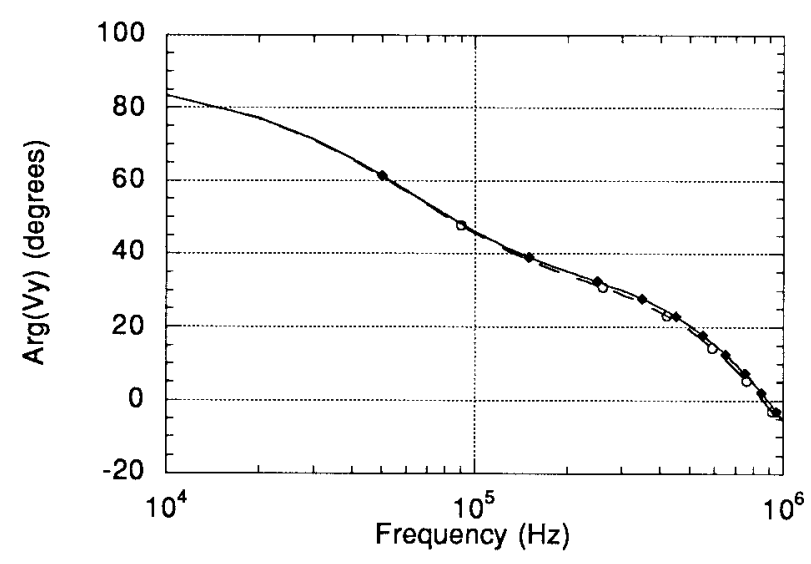

(b)

Fig. 6. Elemental dipole open circuit voltage (continuous line) plotted together with $h_{e} E$ (dotted line).

\section{B. Elemental Dipole}

The voltage across the feed point of the dipole model (Fig. 4) was also calculated and plotted in Fig. 6. The output has the characteristics of the electric field.

The effective length $h_{e}$ for an electrically short cylindrical dipole antenna is given by the expression [16],

$$
h_{e}=\frac{h(\Omega-1)}{2(\Omega-2+\ln 4)}
$$

where the length-thickness parameter $\Omega=2 \ln (h / a), h$ is the total length of the dipole, and the effective radius for a conductor having a square cross section (dimensions $g \times g$ ) is given by [17]

$$
a=0.59 g
$$

This results in $h_{e}=1.76$ if one assumes that the feed point separation is infinitely thin. This separation is, however, one meter wide so the total effective length becomes

$$
h_{e}=2.76 \mathrm{~m}
$$

In Fig. 6, the FDTD voltage and the effective length-electric field product (10) are plotted. Close agreement verifies the use of this structure as a receiving antenna in FDTD investigations. Of importance is the very different shape of the electric field

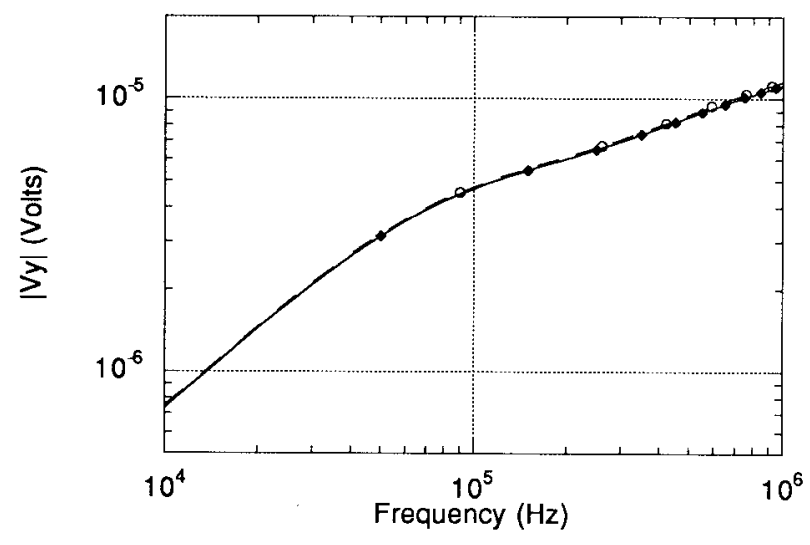

(b)

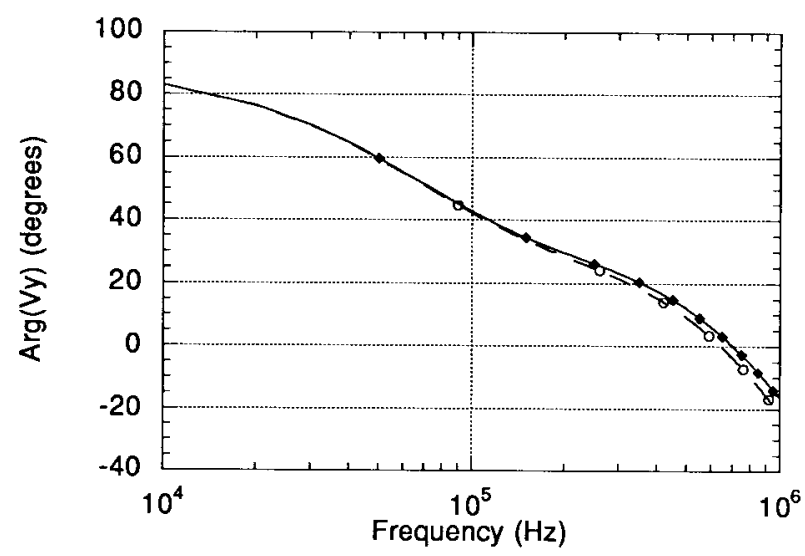

(b)

Fig. 7. Open circuit voltage $V_{y}$ (continuous line) for a staked antenna with no earth penetration, compared with $V_{E}$ (dotted line). Note that the air loop contribution of the voltage has been subtracted from $V_{y}$.

profile across the frequency band when compared with that of the magnetic field shown in Fig. 5. It is because these profiles are so different that one can assess whether the probe is responding to the electric field component, or the magnetic field component at the surface of the earth.

\section{Staked Probe}

In investigating the performance of a staked antenna, it was necessary to use one insulating cell between the horizontal portion of the antenna and the conductive earth. This results in additional magnetic induction in the air portion enclosed in the loop. In order to remove this effect, the open circuit voltage for a partly buried loop was used to quantify this contribution to the voltage. The structure of the staked probe is depicted in Fig. 4. The open circuit voltage measured across the air cell at the feed point of the staked antenna for the staked portion of the probe only was determined by subtracting the voltage from the partially buried loop. The resulting voltage is plotted in Fig. 7, together with the length-electric field product given by (9). The response resembles that from the dipole electric field probe shown in Fig. 6. Note that to obtain the best fit, $h=4 \mathrm{~m}$, which is the mid cell axis separation distance and not the total horizontal length of the staked configuration. 


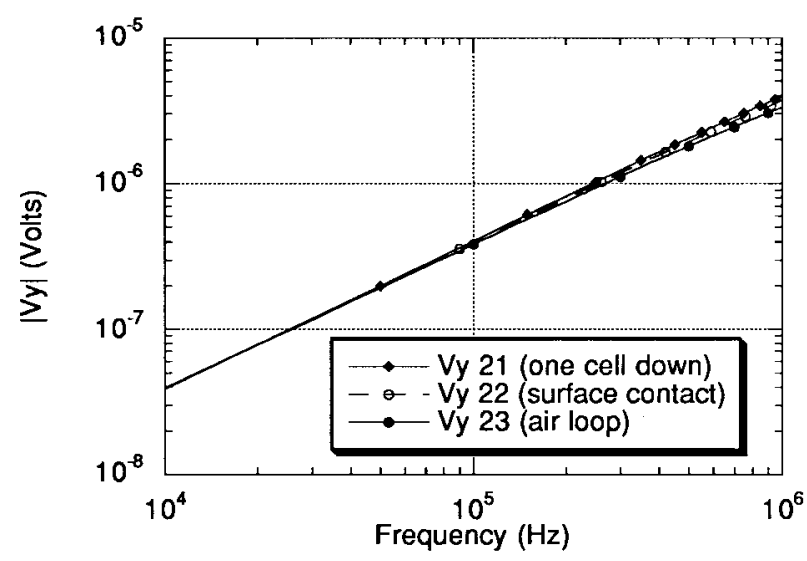

Fig. 8. Open circuit loop voltage showing height and frequency variation.

The excellent agreement shows conclusively that the staked antenna does act as an electric field probe across the frequency range of interest. This includes the situation when the earth depth is effectively infinite and also when the earth is layered.

\section{Partly Buried Loop Antenna}

While the preceding sections show good agreement with theory, there are still some questions which must be addressed. Firstly, the loop antenna gives an open circuit voltage which is much greater than that expected for the open area inside the loop. Secondly, in order to match the staked antenna performance with that predicted, it was necessary to subtract that portion of the response attributed to the air loop above the surface. Given the height gain effect of the horizontal electric field shown in Fig. 3, the possibility of the loop structure having both a "dipole mode" and a "circulating mode" [9] should be re-examined. Fig. 8 shows the open circuit loop voltage for three loop heights; $V_{23}$ corresponds to the loop in air completely, $V_{22}$ corresponds to the bottom of the loop touching the lossy earth plane, and $V_{21}$ corresponds to the partly buried loop shown in Fig. 4. The frequency characteristics of these all indicate that the output voltage is principally related to the magnetic field. At the higher frequencies, there is evidence of a difference between the three plots. This is thought to be the "dipole mode" contribution, as $d E_{y} / d z$ is larger at higher frequencies (Fig. 3).

\section{E. Effective Length Investigation}

Both (9) and (10) have been verified in this paper. Clearly the effective length of the antenna is dependent on the conductivity of the surface of the earth in which the stakes are placed. For example, this argument was introduced for the case of surface impedance measurements over ice-covered regions [9]. The lossy earth plane, shown in Fig. 2, was covered with a one meter deep layer having lower conductivity values. The conductivity of this layer was varied from $10^{-3}$ to $10^{-6} \mathrm{~S} / \mathrm{m}$. The probe was a staked antenna with a subsurface stake length of zero. As with the staked probe investigation, the open circuit voltage was subtracted from the open circuit voltage of the partly-buried loop antenna. From (10), $h_{e}$ is given by the ratio of this voltage and the surface electric field. Fig. 9 shows the

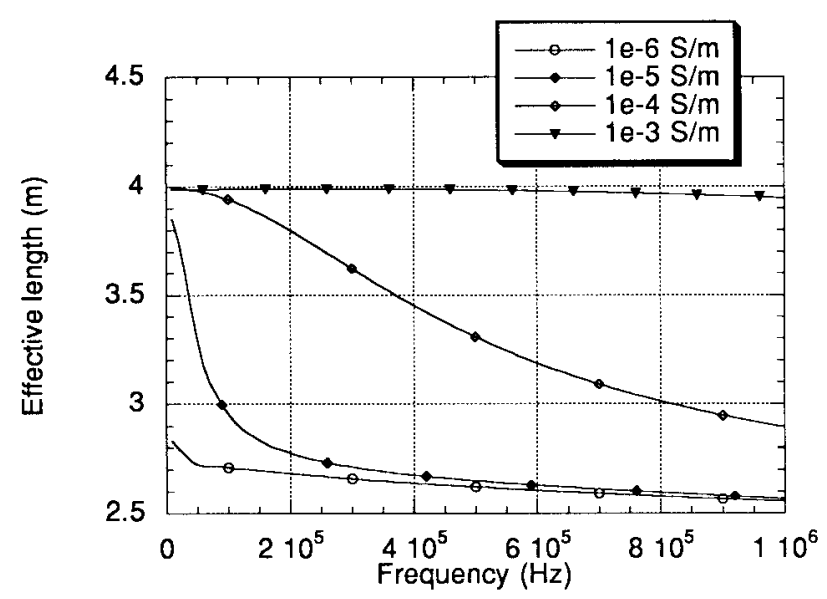

Fig. 9. Variation in the effective length of a staked antenna $h_{e}$ with frequency and upper layer conductivity.

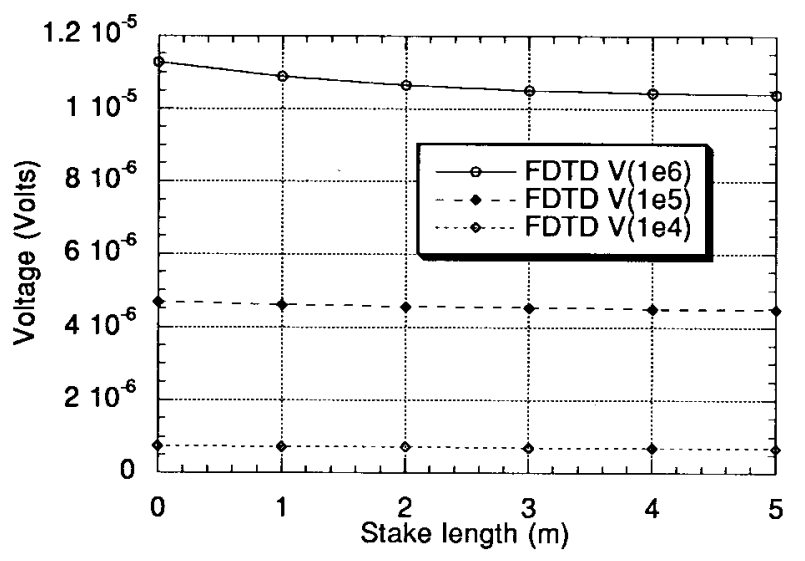

Fig. 10. Variation in staked antenna feed point voltage as a function of stake length measured under the ground.

variation in $h_{e}$ with both frequency and conductivity of the upper layer.

It is clear that there is a transition in the behavior of the staked probe from the one described by (9) to that described by $h_{e}$ in (10). Thus the staked probe has the performance of a loop antenna described by (2) for certain frequencies and upper-layer conductivities, and that of an insulated wire antenna at other frequencies and upper-layer conductivities.

\section{F. Stake Length Investigation}

The result of increasing the length of the stake (measured beneath the earth's surface) was undertaken using the single earth layer model with a conductivity of $0.01 \mathrm{~S} / \mathrm{m}$ and depth of $20 \mathrm{~m}$. The open circuit voltage is given in Fig. 10 for three conductivities as a function of stake length. While there is some change for the case when the stake length approaches one skin depth at $1 \mathrm{MHz}$, the effect at lower frequencies is minimal.

The effect of stake length was also investigated for an earth plane (conductivity $0.01 \mathrm{~S} / \mathrm{m}$ ) covered with more resistive material (conductivity $10^{-4} \mathrm{~S} / \mathrm{m}$ ). Results are shown in Fig. 11, for both 0 and $1 \mathrm{~m}$ stake penetration distances and for upper layer depths of both 1 and $2 \mathrm{~m}$. The effective length of the 


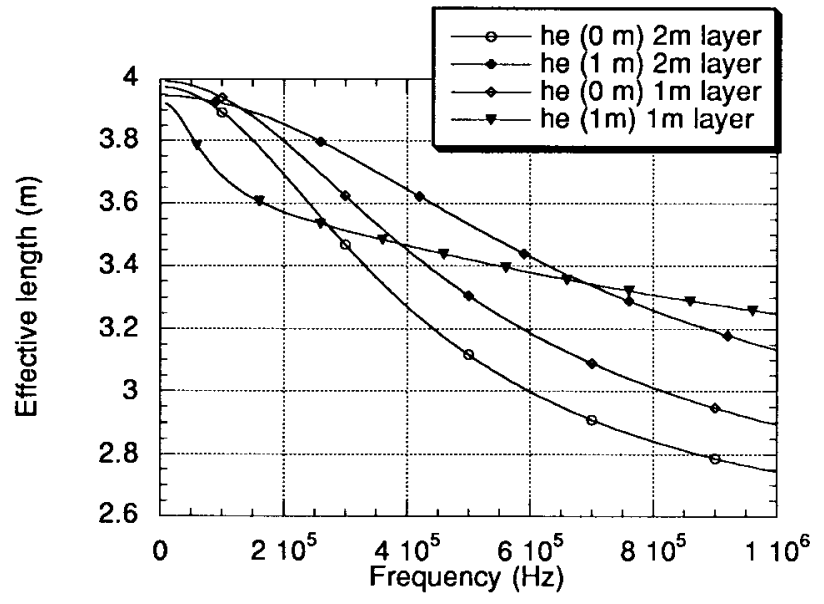

Fig. 11. Variation in the effective length of a staked antenna $h_{e}$ for two different stake lengths ( 0 and $1 \mathrm{~m}$ beneath the surface) for two different earth planes. One consists of a $1 \mathrm{~m}$ layer (conductivity $10^{-4} \mathrm{~S} / \mathrm{m}$ ) overlying a 20 $\mathrm{m}$ deep lossy material (conductivity $0.01 \mathrm{~S} / \mathrm{m}$ ) over a perfect conductor, and the second a $2 \mathrm{~m}$ layer (conductivity $10^{-4} \mathrm{~S} / \mathrm{m}$ ) overlying a $19 \mathrm{~m}$ deep lossy material (conductivity $0.01 \mathrm{~S} / \mathrm{m}$ ) over a perfect conductor.

antenna is very much dependent on the stake length, the depth of the upper layer, and frequency. The case where the stakes penetrate the more resistive layer and make contact with the lower layer has very different characteristics to the other three situations where contact was not made.

\section{Discussion AND CONCLUSIONS}

Fig. 7 clearly demonstrates that a staked probe is an accurate detector of both the amplitude and the phase of the electric field at the surface of a lossy earth plane for both a uniform infinite earth half space and also when the earth has a horizontally layered structure, only for a particular combination of frequency and upper layer conductivity. It is not directly related to the surface magnetic field, but rather to the integrated magnetic field beneath the surface.

The effective length of a staked probe is equivalent to the stake separation distance only when the frequency is sufficiently low and the upper layer conductivity is sufficiently high. If these conditions are not met, the effective length of the probe varies between that of an insulated dipole antenna (approximately one half the length of the probe) and the staked antenna in conductive ground (the length of the probe), and its value is dependent on the conductivity of the upper layer; the depth of the upper layer; the stake penetration length; and, the frequency of measurement.

Given that common earth materials range from $10-10^{-6}$ $\mathrm{S} / \mathrm{m}$, staked earth probes operating at VLF frequencies (10-25 $\mathrm{kHz}$ ) will not give reliable estimates of surface impedance over ground planes where the upper layer is resistive. Examples include ice covered regions, permafrost and dry, sand-covered environments. In the FDTD model used in this investigation, the largest error in assuming (9) is correct will be an underestimate of $63 \%$ of the true value of the surface impedance, which will result in an under-estimate in the apparent resistivity of $40 \%$ of the true value. For more realistic thin wire antennas, the effective length of the probe will reduce to $50 \%$ of the true length, and the apparent resistivity will be reduced to $25 \%$ of the true value. The electrically short insulated antenna accurately reproduces the electric field at the earth's surface; however, the response is approximately one half of the response obtained from the staked earth probe if the upper earth conductivity is sufficiently high.

These results are somewhat similar to those obtained by Zonge and Hughes [12] who showed that if the stake contact resistance is too high, the open circuit voltage is reduced by up to $50 \%$. It must be emphasised that the results presented here reflect the inability of the staked antenna to present consistent electric field measurements in some earth conditions even if there is no contact resistance problem.

\section{ACKNOWLEDGMENT}

D. Thiel wishes to acknowledge the wonderful hospitality of Prof. Mittra and all members of the Electromagnetic Communication Laboratory during his stay. It was greatly appreciated.

\section{REFERENCES}

[1] L. Cagniard, "Basic theory of the magnetotelluric method of geophysical prospecting," Geophysics, vol. 18, no. 3, pp. 605-635, July 1953.

[2] A. N. Tickhonov, "Determination of the electrical characteristics of deep strata of the earth's crust," Dok. Akad. Nauk., USSR, vol. 73, no. 2, pp. 295-297, 1950.

[3] Y. Kato and T. Kikuchi, "On the phase difference of earth current induced by the changes of the earth's magnetic field," Serv. V. Geophys., Science Reports Tohoku Univ., vol. 2, pp. 139-145, 1950.

[4] L. S. Collett and O. G. Jensen, Eds., "Geophysical applications of surface wave impedance measurements," Geolog. Surv. Can., 1982, paper 8115

[5] K. Vozoff, Ed., Magnetotelluric Methods, Society of Exploration Geophysicists. Tulsa, OK: SEG, 1985.

[6] H. P. Patra and K. Mallick, Geosounding Principles 2 Time Varying Geoelectric Soundings. New York: Elsevier, 1980.

[7] X. W. Wu and D. V. Thiel, "Electric field probes for electromagnetic sounding," IEEE Trans. Geosci. Remote Sensing, vol. 27, pp. 24-27, Jan. 1989.

[8] J. R. Wait, "Comments," IEEE Trans. Geosci. Remote Sensing, vol. 27, p. 789, Nov. 1989

[9] X. W. Wu and D. V. Thiel, "Reply to comments," IEEE Trans. Geosci. Remote Sensing, vol. 27, p. 790, Nov. 1989.

[10] J. R. Wait, "Further comments," IEEE Trans. Geosci. Remote Sensing, vol. 27, p. 791, 1989

[11] X. W. Wu and D. V. Thiel, "Reply to comments," IEEE Trans. Geosci. Remote Sensing, vol. 28, pp. 791-792, 1989.

[12] K. L. Zonge and L. J. Hughes, "The effect of electrode contact resistance on electric field measurements," in SEG 55th Conv., Washington, Oct. 1995.

[13] D. V. Thiel and R. Mittra, "Surface impedance modeling using the finite difference time domain method," IEEE Trans. Geosci. Remote Sensing, this issue, pp. 1350-1356.

[14] D. K. Cheng, Field and Wave Electromagnetics, 2nd ed. Reading, MA: Addison Wesley, 1989.

[15] J. Galejs, Terrestrial Propagation of Long Electromagnetic Waves. Oxford: Pergamon Press, 1972.

[16] R. W. P. King, The Theory of Linear Antennas. Cambridge, MA: Harvard Univ. Press, 1956, p. 470.

[17] J. D. Kraus, Antennas. New York: McGraw Hill, 1950.

David V. Thiel (M'81-SM'88), for a photograph and biography, see this issue, p. 1356.

Raj Mittra (S'54-M'57-SM'69-F'71-LF'96), for a photograph and biography, see this issue, p. 1356. 